\title{
Flavonoid and Steroids from Polygonum viscosum
}

\author{
Bidyut Kanti Datta ${ }^{1}$, Tahamina Iasmin ${ }^{1}$ and Mohammad A. Rashid ${ }^{2}$ \\ ${ }^{1}$ Department of Pharmacy, Stamford University Bangladesh, 51, Siddeswari, Dhaka-1217, Bangladesh \\ ${ }^{2}$ Phytochemical Research Laboratory, Department of Pharmaceutical Chemistry, \\ University of Dhaka, Dhaka-1000, Bangladesh
}

Received: May 08, 2016; Accepted: June 28, 2016; Published (Web): July 31, 2016

\begin{abstract}
Repeated chromatographic separation and purification of the $n$-hexane-ethyl acetate $(3: 1)$ extract of aerial parts of Polygonum viscosum provided a flavonoid- ternatin (1) and two steroids- stigmasterol (2) and stigmasta-4,22-dien-3-one (3). The structure of these compounds were determined on the basis of extensive NMR and other spectroscopic techniques and comparison with published data. The identity of steroids 2 and 3 was confirmed by co-TLC with authentic sample.
\end{abstract}

Key words: Polygonum viscosum, Polygonaceae, NMR

\section{Introduction}

Polygonum viscosum Buch-Ham ex D. Don. (Polygonaceae), Bengali name- Biskatali, is an annual herb which is indigenous to Nepal and also widely distributed in Bangladesh, northeast India, Japan and China (Datta et al., 2004). Previous phytochemical studies of different species of Polygonum revealed a variety of secondary metabolites like hydrocarbons, sterols, acetophenones, coumarins, terpenoids and flavonoids (DNP on CD ROM 9.1, 2000; Ezhilan and Neelamegam, 2012; Das and Ganapaty, 2015).

The ethanol extract of whole plant of $P$. viscosum led to the isolation of several flavonoids and terpenoids (Datta et al., 2000, 2001, 2002), some of which showed significant biological activities (Datta et al., 2004). As part of our continuing studies with Polygonum species growing in Bangladesh, we, here in, report the isolation and characterization a flavonoid and two steroid derivatives for the first time.

\section{Materials and Methods}

Plant materials: The aerial parts of Polygonum viscosum Buch-Ham ex D. Don. were collected from Panchori, Chittagong Hill Tracts, Bangladesh in the month of January, 2014 and authenticated by Professor Md. Abul Hassan, Department of Botany, University of
Dhaka, where a voucher specimen has been maintained under the accession number DUSH-764.

Extraction and fractionation: The air dried and coarse powder $(800 \mathrm{gm})$ was extracted with combination of $n$-hexane and ethyl acetate $(3: 1,5.0 \mathrm{~L})$ in a clean flat-bottomed and air tight container for 15 days at room temperature with occasional shaking. The extract was then filtered through a fresh cotton plug followed by Whatman No. 1 filter paper. The filtrate was concentrated with a rotary evaporator at $40-45^{\circ} \mathrm{C}$ and reduced pressure. The crude extract obtained (30 $\mathrm{gm})$ and stored in refrigerator until used.

General experimental procedures: UV (methanol) and IR (KBr) spectra were obtained on Beckman DU 640 and Perkin Elmer FTIR spectrometer, respectively. ${ }^{1} \mathrm{H}$ NMR (400 MHz either $\mathrm{C}_{5} \mathrm{D}_{5} \mathrm{~N}$ or $\mathrm{CDCl}_{3}$ ) and ${ }^{13} \mathrm{C}$ NMR $\left(100 \mathrm{MHz}, \mathrm{C}_{5} \mathrm{D}_{5} \mathrm{~N}\right)$ were acquired on Varian Inova 400 instrument and HR-EIMS have been recorded with a VG Micromass $2 \mathrm{AB}$ mass spectrometer. Vacuum Liquid Chromatography (VLC) column was run on Kiselgel $60 \mathrm{H}$ (100-200 mesh) and analytical and preparative thin layer chromatography (TLC and PTLC) were performed on glass plates coated with silica gel (Kiselgel $60 \mathrm{PF}_{254}$ ). Solvent systems ranging from non-polar to polar were used. UV light $(254 \mathrm{~nm}$ and $365 \mathrm{~nm})$ and vanillin $/ \mathrm{H}_{2} \mathrm{SO}_{4}$ reagents (1:100) (Stahl, 1966) were used to visualize the spots as Correspondence to: Bidyut Kanti Datta; Phone: +8801715124177; E-mail: dattabidyut@ yahoo.com 
appropriate. Other reagents and chemicals were of analytical grade.

\section{Properties of isolated compounds}

Properties of ternatin (1): Yellow crystals, m.p. $217{ }^{\circ} \mathrm{C}$; Found $\mathrm{M}^{+}$374.1001, $\mathrm{C}_{19} \mathrm{H}_{18} \mathrm{O}_{8}$, requires 374.1002; UV $\lambda_{\max }(\mathrm{MeOH}) \mathrm{nm}: 254,272,336,368$; IR $v_{\max }(\mathrm{KBr}) \mathrm{cm}^{-1}: 3350,2940,1650,1595,1460,1360$, $1265,1160,1045,920,820 ;{ }^{1} \mathrm{H}$ NMR $(400 \mathrm{MHz}$, $\left.\mathrm{C}_{5} \mathrm{D}_{5} \mathrm{~N}\right): 13.01(1 \mathrm{H}, \mathrm{s}, \mathrm{OH}-5), 8.07(1 \mathrm{H}, \mathrm{dd}, J=8.1,1.7$ $\left.\mathrm{Hz}, \mathrm{H}-6^{\prime}\right), 8.01\left(1 \mathrm{H}, \mathrm{d}, J=1.7 \mathrm{~Hz}, \mathrm{H}-2^{\prime}\right), \delta 7.37(1 \mathrm{H}, \mathrm{d}$
$J=8.1 \mathrm{~Hz}, \mathrm{H}-5), 6.71(1 \mathrm{H}, \mathrm{s}, \mathrm{H}-6), 4.04(3 \mathrm{H}, \mathrm{s}, \mathrm{OMe}-$ 3), $4.03(3 \mathrm{H}, \mathrm{s}, \mathrm{OMe}-8), 3.91$ (3H, s, OMe-3'), 3.85 $(3 \mathrm{H}, \mathrm{s}, \mathrm{OMe}-7) ;{ }^{13} \mathrm{C} \mathrm{NMR}\left(100 \mathrm{MHz}, \mathrm{C}_{5} \mathrm{D}_{5} \mathrm{~N}\right): 156.9$ (C-2), 139.2 (C-3), 179.8 (C-4), 158.3 (C-5), 96.6 (C6), 159.4 (C-7), 129.8 (C-8), 149.3 (C-9), 106.2 (C-10), 122.4 (C-1'), 112.9 (C-2'), 149.1 (C-3'), 152.2 (C-4'), 117.4 (C-5'), 123.9 (C-6'), 60.4 (OMe-3), 56.9 (OMe7), 61.9 (OMe-8), 56.3 (OMe-3'); EIMS, $m / z$ (rel. int.): $374[\mathrm{M}]^{+}$(64), 373 (5), 361 (2), 360 (20), 359 (100), 331 (3), 153 (6), 95 (6).<smiles>CCC(/C=C/C(C)C1CC2C3CCC4=CC(=O)CCC4(C)C3CCC2(C)CC1C(C)/C=C/C(CC1CC2C3CCC4=CC(C)CCC4(C)C3CCC2(C)C1)C(C)C)C(C)C</smiles>

Properties of stigmasterol (2): White needles, m.p. 126-128 ${ }^{\circ} \mathrm{C}$; Found $\mathrm{M}^{+} 412.7051, \mathrm{C}_{29} \mathrm{H}_{48} \mathrm{O}$, requires 412.7053; IR $v_{\max }(\mathrm{KBr}) \mathrm{cm}^{-1}: 3450,2940,2820,2450$, 1640, 1450, 1360, 1040, 850; ${ }^{1} \mathrm{H}$ NMR (400 MHz, $\left.\mathrm{CDCl}_{3}\right): \delta 5.37(1 \mathrm{H}, \mathrm{m}, \mathrm{H}-6), 5.16(1 \mathrm{H}, \mathrm{dd}, J=15.2$, $8.0 \mathrm{~Hz}, \mathrm{H}-22), 5.03(1 \mathrm{H}, \mathrm{dd}, J=15.2,7.2 \mathrm{~Hz}, \mathrm{H}-23)$, $3.55(1 \mathrm{H}, \mathrm{m}, \mathrm{H}-3), 1.03(3 \mathrm{H}, \mathrm{s}, \mathrm{Me}-13), 0.90(3 \mathrm{H}, \mathrm{d}, J=$ $6.8 \mathrm{~Hz}, \mathrm{Me}-20), 0.83(3 \mathrm{H}, \mathrm{d}, J=6 \mathrm{~Hz}, \mathrm{Me}-25), 0.81$ $(3 \mathrm{H}, \mathrm{t}, J=6.5 \mathrm{~Hz}, \mathrm{Me}-28), 0.69$ (3H, s, Me-10); EIMS, $\mathrm{m} / z$ (rel. int.): 412 (19), 271 (14), 213 (29), 173 (14), 133 (30), 91 (46), 55 (73), 43 (100).

Properties of stigmasta-4,22-dien-3-one (3): White gum, FABMS $m / z$ : Found $411.6971[\mathrm{M}+\mathrm{H}]^{+}, \mathrm{C}_{29} \mathrm{H}_{46} \mathrm{O}$, requires $411.6974\left(\mathrm{C}_{29} \mathrm{H}_{46} \mathrm{O}+\mathrm{H}\right)$; IR $v_{\max }$ (Film) $\mathrm{cm}^{-1}$ : 2928, 2852, 1680, 1672, 1649, 1450; ${ }^{1} \mathrm{H}$ NMR (400 $\left.\mathrm{MHz}, \mathrm{CDCl}_{3}\right): \delta 5.73(1 \mathrm{H}, \mathrm{s}, \mathrm{H}-4), 5.16(1 \mathrm{H}, \mathrm{dd}, J=$ $15.2,8.0 \mathrm{~Hz}, \mathrm{H}-22), 5.05(1 \mathrm{H}, \mathrm{dd}, J=15.2,7.2 \mathrm{~Hz}, \mathrm{H}-$ 23), 1.19 (3H, s, Me-10), $0.93(3 \mathrm{H}, \mathrm{d}, J=6.8 \mathrm{~Hz}, \mathrm{Me}-$ 20), 0.86 (3H, d, $J=6 \mathrm{~Hz}$, Me-25), $0.82(3 \mathrm{H}, \mathrm{t}, J=6.5$ $\mathrm{Hz}, \mathrm{Me}-28), 0.73$ (3H, s, Me-13).

\section{Results and Discussion}

The HR-EMIS of the yellow crystalline compound (1) gave a molecular ion peak at $\mathrm{m} / \mathrm{z}, 374$ appropriate for the molecular formula, $\mathrm{C}_{19} \mathrm{H}_{18} \mathrm{O}_{8}$. The ${ }^{1} \mathrm{H}$ NMR spectrum showed an aromatic A-ring proton signal for H-6 at $\delta 6.71$, a C-5 hydroxyl at 13.17 , four methoxyl resonances between 3.85 4.04 and three B-ring aromatic proton signals typical for a 3',4'-dioxygenated flavonols (Butterham and Highet, 1964). The meta coupled (H-2') and ortho-meta coupled (H-6') appeared at $\delta 8.01 \sim 8.07$ while the ortho coupled $\mathrm{H}-5^{\prime}$ signal was obserevd at $\delta$ 7.37. The ${ }^{13} \mathrm{C}$ spectrum showed 19 carbons including four methoxyls, four methines, one carbonyl and the 10 quaternary carbon signals which further supported the molecular formula.

The correlations between protons with carbons were revealed by HC-COBI and HMBC experiments (Table 1). Among four methoxyls, the deshielded one at $\delta 4.04$ was attached to $\mathrm{C}-3$ as its connectivity was obserevd to the oxygenated quaternary at $\delta$ 139.2, which is the typical resonance for C-3 in 3-methoxy flavonols. The remaining three methoxyls at $\delta 3.85$, 
4.03 and 3.91, demonstrated connectivity over three bonds to the oxygenated quaternary carbons at $\delta 159.4$, 129.8 and 149.1, respectively. These carbons were also identified as C-7, C-8 and C-3' by the ${ }^{2} J$ or ${ }^{3} J$ correlations from $\mathrm{H}-6$ and $\mathrm{H}-5$, respectively. On the basis of these data, compound $\mathbf{1}$ was identified as 5,4'dihydroxy-3,7,8,3'-tetramethoxyflavonol (ternatin), a flavonoid previously isolated from Begonia glabra (Ensemeyer and Langhammer, 1982)

Compound 2 was obtained as white needle shaped crystals and the HR-EMIS showed the molecular ion peak at $\mathrm{m} / \mathrm{z} 412$ solving the molecular formula as
$\mathrm{C}_{29} \mathrm{H}_{48} \mathrm{O}$. The ${ }^{1} \mathrm{H}$ NMR spectrum revealed a multiplet of one proton intensity at $\delta 3.55$, the position and multiplicity of which was indicative of $\mathrm{H}-3$ of the steroidal nucleus. The typical signal for H-6 of the steroidal skeleton was evident from a multiplet at $\delta 5.37$ that integrated for one proton. The olefinic proton at $\mathrm{H}$ 22 and H-23 appeared as characteristics downfield signals $\delta 5.16$ and 5.03 respectively. The spectrum further revealed signals for six methyl groups. On this basis, compound $\mathbf{2}$ was characterized as stigmasterol (Ikan, 1991), which was further confirmed by co-TLC with authentic stigmasterol.

Table $1 .{ }^{1} \mathrm{H}-{ }^{13} \mathrm{C}$ correlations for ternatin (1) as determined by $\mathrm{HC}-\mathrm{COBI}$ and HMBC experiments.

\begin{tabular}{llll}
\hline Protons & \multicolumn{3}{l}{${ }^{1} \mathrm{H}^{-13} \mathrm{C}$ correlation } \\
\hline & ${ }^{1} J$ & ${ }^{3}$ & \\
\hline OMe & $60.4(\mathrm{OMe}-3)$ & $139.2(\mathrm{C}-3)$ & \\
OMe & $56.9(\mathrm{OMe}-7)$ & $159.4(\mathrm{C}-7)$ & \\
$\mathrm{OMe}$ & $61.9(\mathrm{OMe}-8)$ & $129.8(\mathrm{C}-8)$ & $158.3(\mathrm{C}-5)$ \\
$\mathrm{OMe}$ & $56.3\left(\mathrm{OMe}-3^{\prime}\right)$ & $149.1\left(\mathrm{C}-3^{\prime}\right)$ & $159.4(\mathrm{C}-7), 158.3(\mathrm{C}-5)$ \\
$\mathrm{OH}-5$ & - & $106.2(\mathrm{C}-10), 96.6(\mathrm{C}-6)$ & $149.1\left(\mathrm{C}-3^{\prime}\right)$ \\
$\mathrm{H}-6$ & $96.6(\mathrm{C}-6)$ & $129.8(\mathrm{C}-8), 106.2(\mathrm{C}-10)$ & $152.3\left(\mathrm{C}-4^{\prime}\right)$ \\
$\mathrm{H}-2^{\prime}$ & $112.9\left(\mathrm{C}-2^{\prime}\right)$ & $156.9(\mathrm{C}-2), 152.2(\mathrm{C}-4)$ & \\
$\mathrm{H}-5^{\prime}$ & $117.4\left(\mathrm{C}-5^{\prime}\right)$ & $149.1\left(\mathrm{C}-3^{\prime}\right), 122.4\left(\mathrm{C}-1^{\prime}\right)$ & \\
$\mathrm{H}-6^{\prime}$ & $123.9\left(\mathrm{C}-6^{\prime}\right)$ & & \\
\hline
\end{tabular}

Compound $\mathbf{3}$ was obtained as white gum. The FABMS displayed the pseudo-molecular ion peak at $m / z \quad 411.6971 \quad[\mathrm{M}+\mathrm{H}]^{+}$establishing its molecular formula as $\mathrm{C}_{29} \mathrm{H}_{46} \mathrm{O}$. The ${ }^{1} \mathrm{H}$ NMR spectrum of 3 revealed a downfield signal for olefinic proton at $\delta$ 5.73 , typical for $\mathrm{H}-4$ of a steroidal nucleus containing a ketone group at $\mathrm{C}-3$ position. The side chain olefinic protons at $\mathrm{H}-22$ and $\mathrm{H}-23$ appeared as characteristic downfield resonances at $\delta 5.16$ and 5.05, respectively. The spectrum also showed a total of six methyl group signals, including three doublets, a triplet and two singlets. Thus, compound $\mathbf{3}$ was charecterized as stigmasta-4,22-dien-3-one (SDBS, 2000). Its identity was substantiated by co-TLC with authentic sample.

\section{References}

Buckingham, J. Eds. 2004. Dictionary of Natural Products. Version 9.2 on CD-ROM. Chapman \& Hall/ CRC Press, London, New York.

Das, S. and Ganapaty, S. 2015. Phytochemical evaluation of roots of Polygonum viscosum Buch-ham. Indian J. Pharm. Sci. 77, 352-356.

Datta, B.K., Datta, S.K., Rashid, M.A., Nash, R.J. and Sarker S.D. 2000. A sesquiterpene acid and flavonoids from Polygonum viscosum. Phytochemistry 54, 201-205.

Datta, B.K., Datta, S.K. and Sarker, S.D. 2000. Quercetin-3$O$-(6"-galloyl)- $\beta$-D-galactoside from Polygonum viscosum (Polygonoceae). Biochem. Syst. Ecol. 28, 805807.

Datta, B.K., Datta, S.K. and Sarker, S.D. 2000. Quercetin-3$O$-(6"-galloyl)- $\beta$-D-galactopyranoside from Polygonum viscosum. Fitoterapia 71, 459-460. 
Datta, B.K., Rashid, M.A., Datta, S.K. and Sarker, S.D. 2001. Viscozulenic acid: a novel sesquiterpene acid from Polygonum viscosum. Pharm. Biol. 39, 198-201.

Datta, B.K., Rashid, M.A., Kundu, J.K., Rouf, A.S.S., Sarker S.D. and Datta, S.K. 2001. Isolation and structure elucidation of viscoazucine: a novel sesquiterpene acid from Polygonum viscosum. Die Pharmazie 56, 578-579.

Datta, B.K., Datta, S.K., Chowdhury, M.M., Khan, T.H., Rashid, M.A., Nahar, L. and Sarker, S.D. 2004. Analgesic, anti-inflammatory and CNS depressant activities of sesquiterpenes and a flavonoid glycoside from Polygonum viscosum. Die Pharmazie 59, 222-225.

Ensemeyer, M., and Langhammer, L. 1982. Two lipophilic flavonoids from Begonia glabra. Planta Med. 46, 254-255.
Ezhilan, B.P. and Neelamegam, R. 2012. GC-MS analysis of phytocomponents in the ethanol extract of Polygonum chinense L. Pharmacog. Res. 4, 11-14.

Ikan, R. 1991. Natural Product - A Laboratory guide, $2^{\text {nd }}$ Eds, Academic press, New York, pp. 1-22.

SDBS: Integrated Spectral Database System for Organic Compounds, 2000, SDBS number J 997, National Institute of Materials and Chemical Research, Tsukuba, Japan, available online at http://www.aist.go.jp/ RIODB/SDBS/menu-e.html.

Stahl, E. 1966. Thin Layer Chromatography, 2 $2^{\text {nd }}$ Eds., Springer Verlag Berlin, Heidelberg, New York, pp. 855904. 